Supplementary Information: Revealing Factors Influencing the

\title{
Operational Stability of Perovskite Light Emitting Diodes
}

Jonathan H. Warby, Bernard Wenger, Alexandra J. Ramadan, Robert D. J. Oliver, Harry C. Sansom,

Ashley R. Marshall and Henry J. Snaith*

Clarendon Laboratory, Department of Physics, University of Oxford, Oxford, OX1 3PU, UK

a)

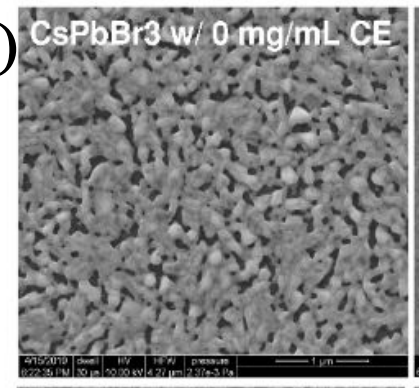

$\mathrm{CsPbBr} 3 \mathrm{w} / 4 \mathrm{mg} / \mathrm{mL} \mathrm{CE}$
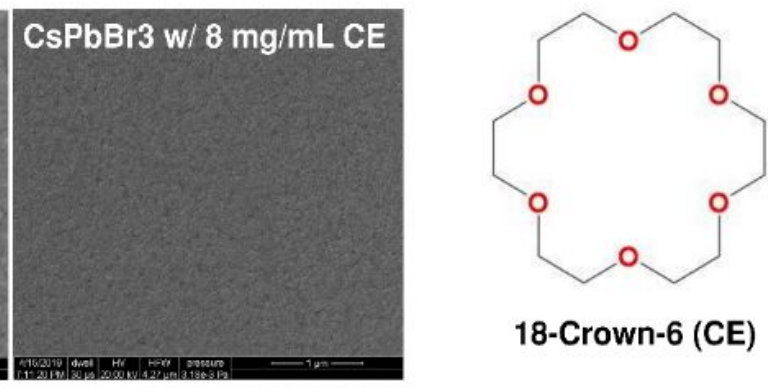

18-Crown-6 (CE)

及)
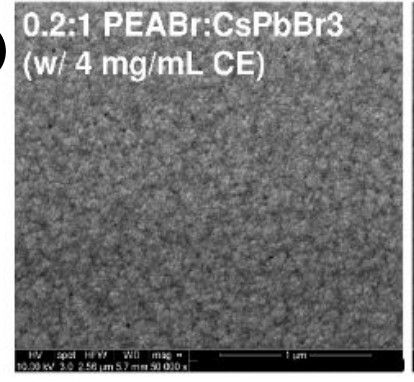

0.4:1 PEABr:CsPbBr3

(w/ $4 \mathrm{mg} / \mathrm{mL}$ CE)
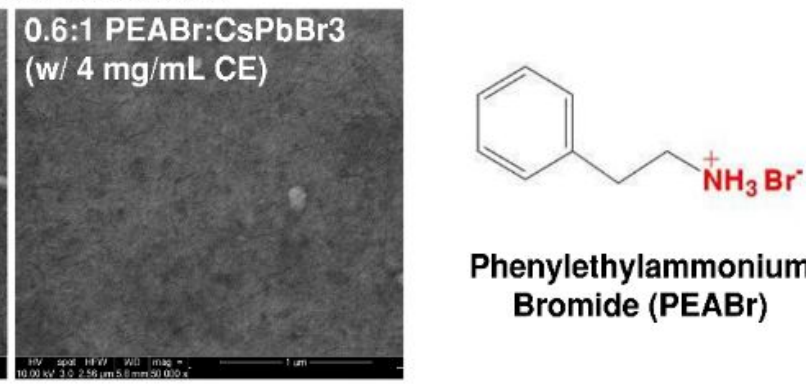

Phenylethylammonium

Bromide (PEABr)

Figure S1. a) The effect of increasing 18-crown-6 concentration on film morphology

and its structure. 18-crown-6 is part of the crown ether (CE) family which is known to improve solubility of salts in various solvents through the chelate effect. ${ }^{1-3}$ Addition of 18-crown-6 has a marked impact on the film morphology. We observe at a concentration of $4 \mathrm{mg} / \mathrm{mL}$ a significant reduction in grain size and improvement in 
surface coverage which agrees with the report from Ban et al. At $8 \mathrm{mg} / \mathrm{mL}$ the grain size is reduced further confirming the strong effect of CE on the film morphology; b) The effect of PEABr on film morphology and the chemical structure of $\mathrm{PEABr}$, all of the films have $4 \mathrm{mg} / \mathrm{mL}$ of $18-$ crown- 6 in the precursor solution. Additon of PEABr has a strong effect on the film morphology causing even further decrease in grain size until we cannot easily resolve it by SEM.

\begin{tabular}{|l|l|l|l|l|l|l|}
\hline & $\begin{array}{l}\text { CsPbBr } \\
3\end{array}$ & $(\mathrm{PEABr})_{0.1}$ & $(\mathrm{PEABr})_{0.2}$ & $(\mathrm{PEABr})_{0.4}$ & $(\mathrm{PEABr})_{0.6}$ & $(\mathrm{PEABr})_{1.0}$ \\
\hline CsBr & 48.5 & 46.9 & 45.3 & 42.56 & 38.4 & 33.8 \\
\hline $\mathrm{PbBr2}$ & 83.6 & 80.8 & 78.2 & 73.402 & 66.3 & 58.3 \\
\hline $\mathrm{PEABr}$ & 0 & 4.45 & 8.6 & 16.2 & 27.4 & 40.1 \\
\hline crown & 4 & 4 & 4 & 4 & 4 & 4 \\
\hline
\end{tabular}

Table S1. Masses of precursors used to make solutions for study. All masses are in $\mathrm{mg} / \mathrm{mL}$. We sought to maintain the same film thickness (measured as $40 \mathrm{~nm}$ for all 
samples) so that the electrical field would be the same in all the devices at constant bias. As such, the total weight of salts inside the solution was fixed at $12.3 \mathrm{wt} \%$

\section{Supplementary Note 1:}

In previous work we have shown that broadening of the excitonic absorption in metal halide perovskites is related to increased energetic disorder which normally correlates with a decrease in optoelectronic performance. ${ }^{4,5} \mathrm{Here}$, we compare the full width half maxima (FWHM) of the photoluminescence spectra with the linear slope of the absorption onset in figure S2. We see that the decrease in slope of absorption and increase in FWHM of the PL spectra follow the same trends which is consistent with emission occurring from a broader distribution of excitonic energies. Counterintuitively, as the spectra broaden, we observe an increase in PLQY. We believe that this is due to a high polydispersity in grain size (which is a form of disorder), where the emissive domains are in the quantum confinement regime, as indicated by the blue shift of the peak wavelength with respect to neat $3 \mathrm{D} \mathrm{CsPbBr}$. Hence, the increased FWHM does not necessarily indicate that emission is from a 
single homogeneous material with a high degree of energetic disorder, but that the emission is from an ensemble of poly-disperse nanoscale crystallite domains. This is consistent with our fabrication process which is not optimized to control of polydispersity. The polydispersity of the system should not be deleterious to the optoelectronic properties of the system, as indicated by the improved PLQY in the presence of PEABr. However, we should highlight that the broadening of the FWHM is disadvantageous for maximizing color purity for use in a display. 


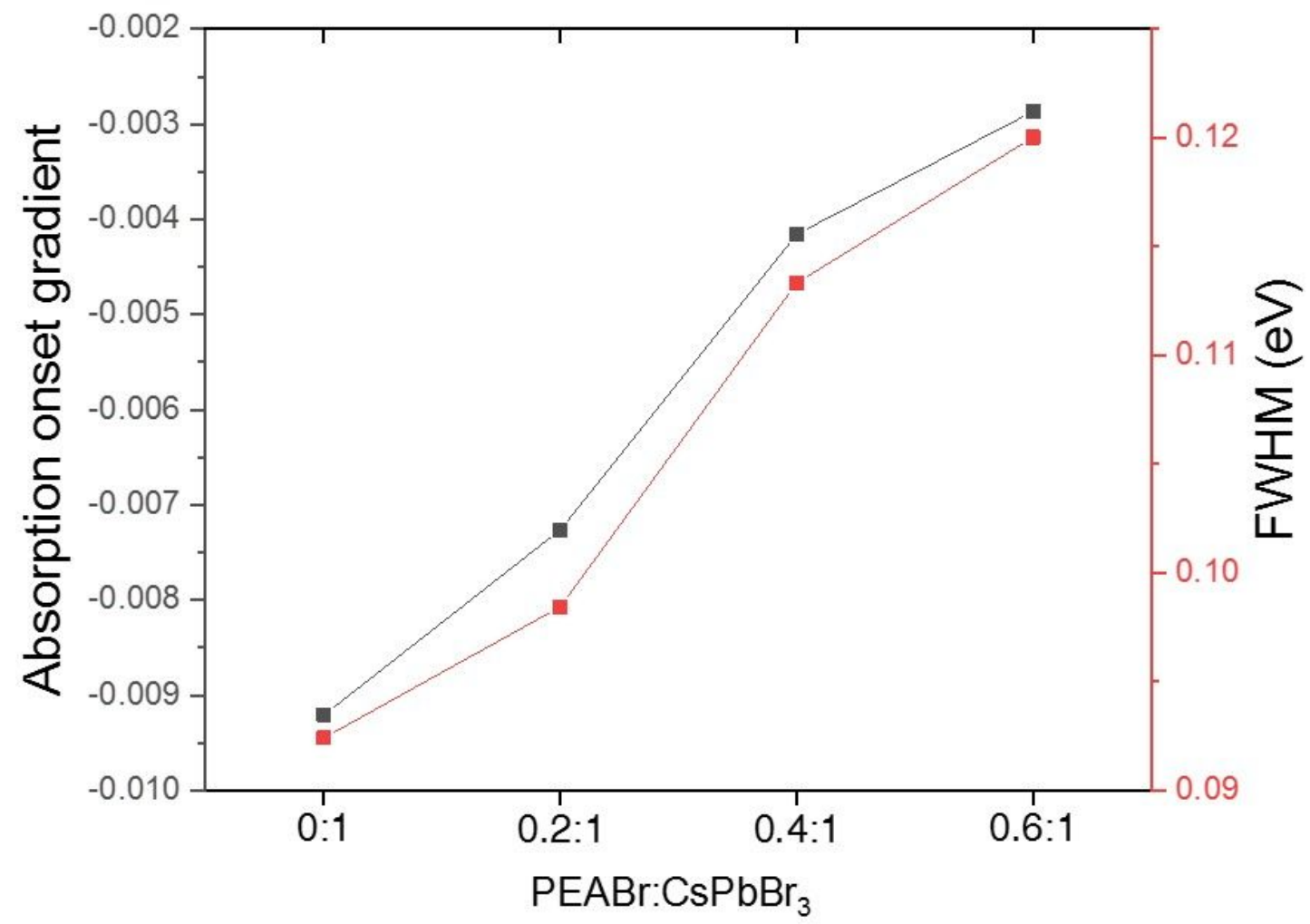

Figure S2. Gradient of Absorption onset and full width half maxima of PL spectra v $\mathrm{PEABr}$ concentration in $\mathrm{CsPbBr}_{3}$. We see that they follow the same trend and thus conclude that they are both linked to increase in energetic disorder 
a)

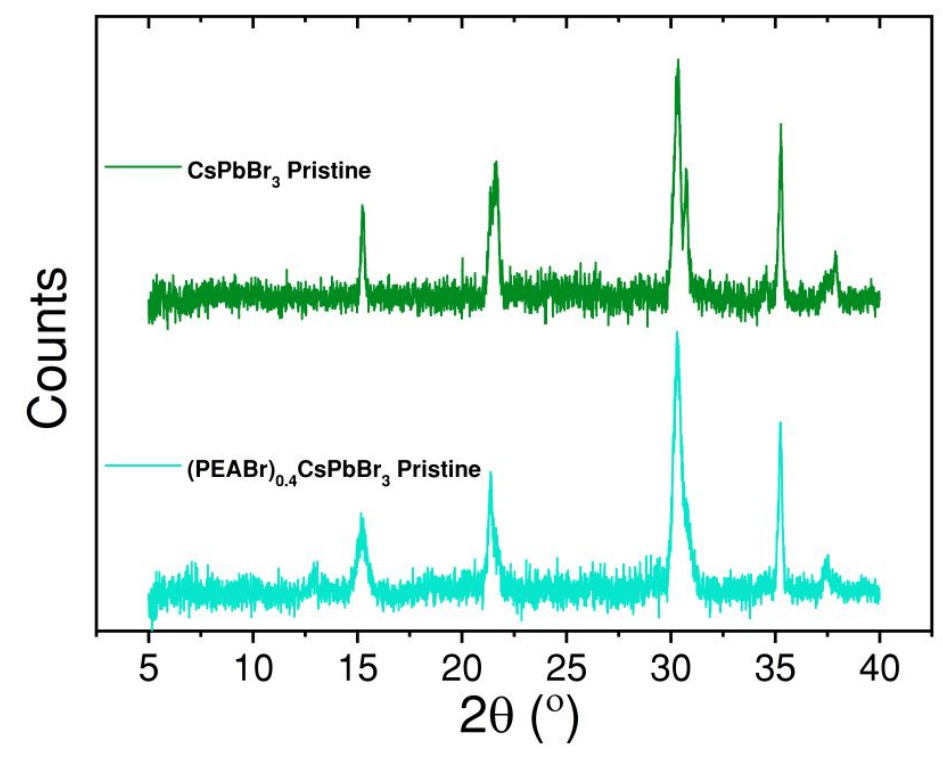

b) $\quad \frac{\mathrm{Cs} \mathrm{PbBr}_{3}}{\mathrm{R}_{3}} \frac{\mathrm{PXRD} \text { peak assignments }}{\mathrm{Cs}_{\mathrm{PbBr}} \text { peaks }}$

Green are ITO peaks

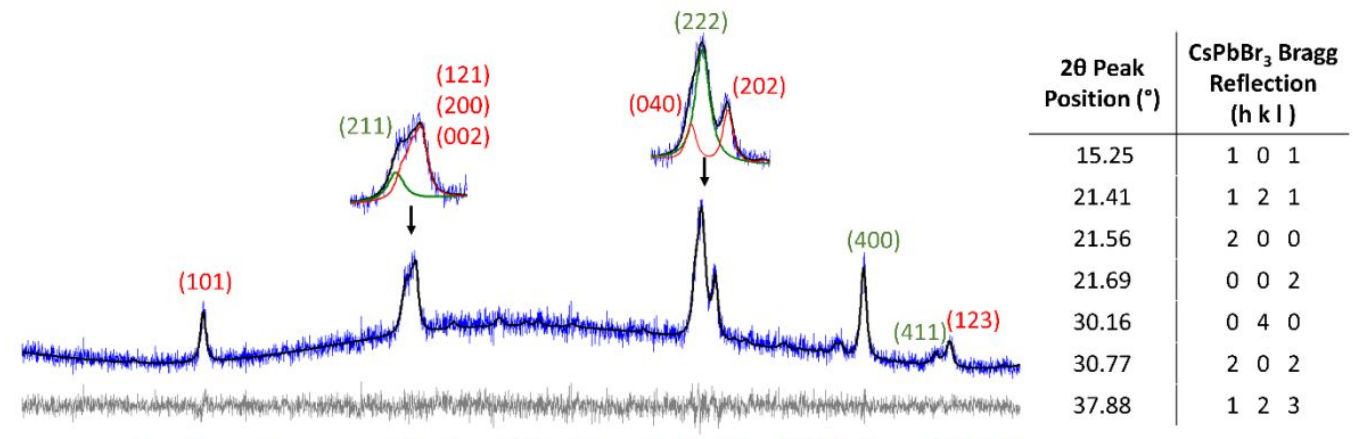

(PEABr) $+\mathrm{CSPbBr}_{3}$ PXRD peak assignments

Red are $\mathrm{CsPbB}_{3}$ peaks

n)

Green are ITO peaks

\begin{tabular}{c|ccc}
$\begin{array}{c}\text { 20 Peak } \\
\text { Position ( }\end{array}$ & $\begin{array}{c}\mathrm{CsPbBr}_{3} \text { Bragg } \\
\text { Reflection } \\
\text { (h }\end{array}$ & I)
\end{tabular}

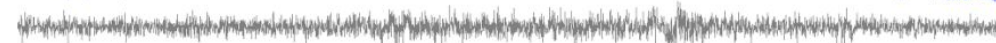


- The PXRD scans have been aligned by aligning ITO (400) peak

d) - The peak positions, and therefore lattice parameters, are the same within error (the numbers in the parentheses correspond to $1 \sigma$ error)

- The (PEA)Br $+\mathrm{CsPbBr}_{3}$ has a much higher FWHM.
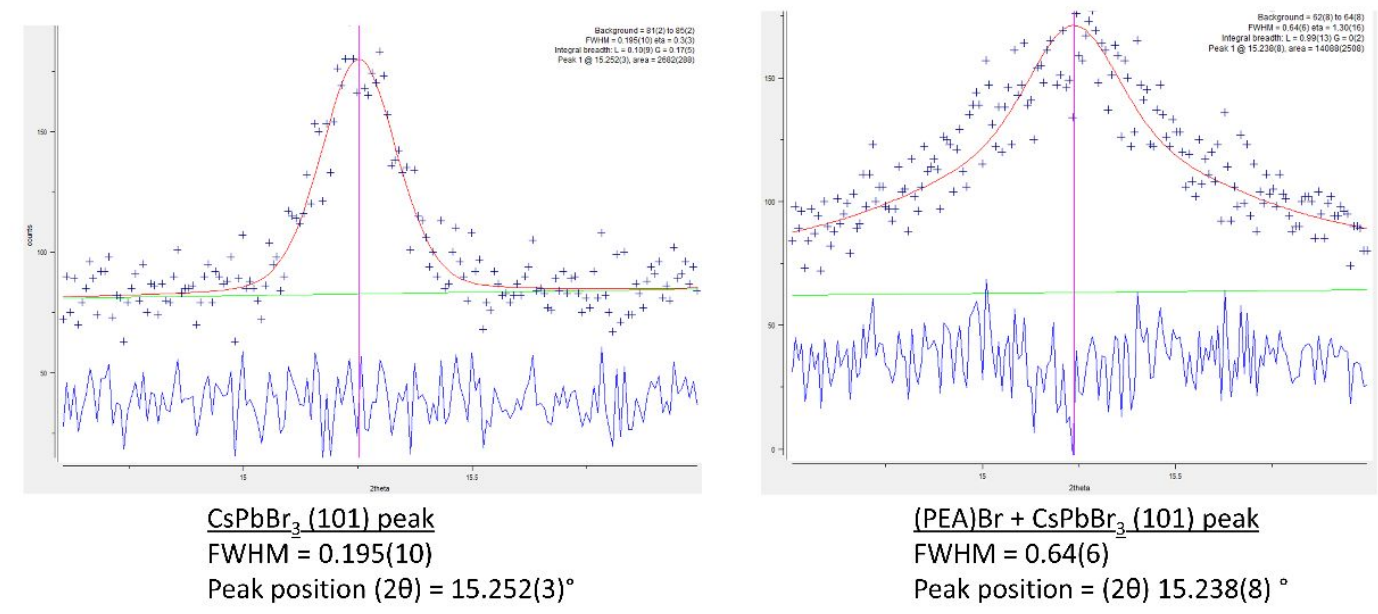

Figure S3. a) XRD data for films of $\mathrm{CsPbBr}_{3}$ and $(\mathrm{PEABr})_{0.4} \mathrm{CsPbBr}_{3}$. It is worth noting that $(\mathrm{PEABr})_{0.4} \mathrm{CsPbBr}{ }_{3}$ does not show any diffraction peaks from lower dimensional layered perovskite; b) Peak assignments of $\mathrm{CsPbBr}_{3}$; c) Peak assignments of $\left.(\mathrm{PEABr})_{0.4} \mathrm{CsPbBr}_{3} ; \mathrm{d}\right)$ comparison of the FWHM of $(\mathrm{PEABr})_{0.4} \mathrm{CsPbBr}_{3}$ and $\mathrm{CsPbBr}_{3}$ (101) peaks, $(\mathrm{PEABr})_{0.4} \mathrm{CsPbBr}_{3}$ has a $\mathrm{FWHM} 3 \times$ wider than $\mathrm{CsPbBr}{ }_{3}$ 


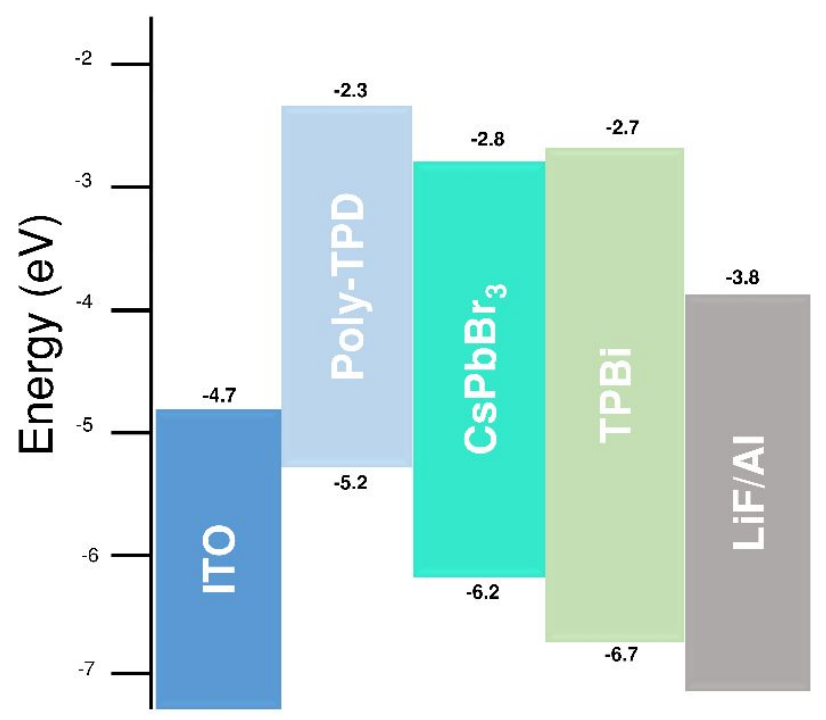

Figure S4. Energy level diagram of perovskite LEDs in this study. Note that this is only with respect to vacuum for isolated films and does not necessarily reflect the precise energy level alignment in the constructed LEDs.

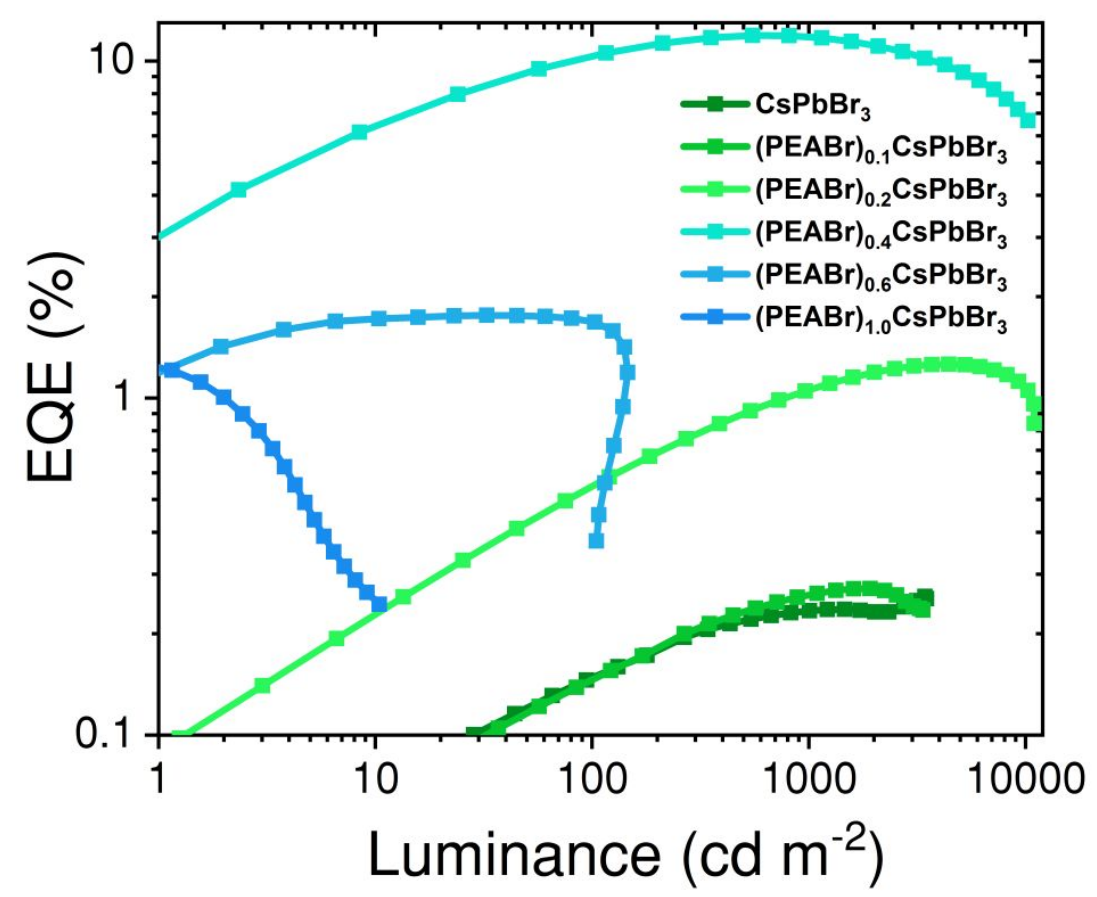


Figure S5. Typical Luminance vs $\mathrm{EQE}_{\mathrm{EL}}$ curves for all compositions of LED in this

\begin{tabular}{|c|c|c|c|c|}
\hline Emitter material & Device structure & $\begin{array}{l}\text { Max } \\
E Q E_{E L}\end{array}$ & $\begin{array}{l}\text { Operationa } \\
\text { / Stability }\end{array}$ & $\begin{array}{l}\text { Operating } \\
\text { conditions }\end{array}$ \\
\hline $\mathrm{CsPbBr}_{3}$ from $\mathrm{CsTFA}$ & $\begin{array}{l}\text { ITO/PEDOT:PSS/Perov/TPBi/LiF/A } \\
\text { । }\end{array}$ & $10.50 \%$ & $\mathrm{t} 50>250 \mathrm{~h}$ & $\begin{array}{l}\text { Initial } \\
\text { luminance = } \\
100 \mathrm{~cd} \mathrm{~m}^{-2}\end{array}$ \\
\hline $\begin{array}{l}\mathrm{CsPbBr}_{3} \\
\text { (evaporated) }\end{array}$ & $\begin{array}{l}\text { ITO/PEDOT:PSS/Perov/TmPyPB/L } \\
\text { iq/Al }\end{array}$ & $1.55 \%$ & $\mathrm{t} 50=38 \mathrm{~min}$ & $\begin{array}{l}\text { Initial } \\
\text { luminance = } \\
200 \mathrm{~cd} \mathrm{~cm}^{-2}\end{array}$ \\
\hline $\mathrm{CsPb}_{2} \mathrm{Br}_{5} \mathrm{Cs} \mathrm{PbBr}_{3}^{8}$ & $\begin{array}{l}\text { ITO/PEDOT:PSS/Perov/TPBi/LiF/A } \\
\text { । }\end{array}$ & $1.10 \%$ & $\mathrm{t} 50=\sim 6$ hours & $10 \mathrm{~mA} \mathrm{~cm}^{-2}$ \\
\hline $\mathrm{CsPbBr}_{3}: \angle i B r^{\ominus}$ & $\begin{array}{l}\text { ITO/NiOx/TFB/PVK/Perov/TPBi/LiF } \\
\text { IAl }\end{array}$ & $16.2 \%$ & $\mathrm{t} 50=21$ hours & $\begin{array}{l}\text { Initial } \\
\text { luminance = } \\
100 \mathrm{~cd} \mathrm{~m}^{-2}\end{array}$ \\
\hline $\mathrm{CsPbBr}_{3}: \mathrm{PEO}^{10}$ & $\begin{array}{l}\text { ITO/PEDOT:PSS/Perov/TPBi/LiF/A } \\
\text { I }\end{array}$ & $4.76 \%$ & $\mathrm{t} 82=80 \mathrm{~h}$ & $\begin{array}{l}\text { Initial } \\
\text { luminance = } \\
1000 \mathrm{~cd} \mathrm{~m}^{-2}\end{array}$ \\
\hline $\mathrm{CsPbBr}_{3}^{11}$ & ITO/BufHIL/Perov/TPBi/LiF/AI & $1.37 \%$ & $\mathrm{~L} 50 \approx 34 \mathrm{~min}$ & $50 \mathrm{~cd} \mathrm{~cm}^{-2}$ \\
\hline $\mathrm{CsPbBr}_{3}: \mathrm{CsBr^{2 }}$ & $\begin{array}{l}\text { ITO/PEDOT:PSS/MHP/ } \\
\text { B3PYMPM/Cs2CO3 /AI }\end{array}$ & $0.15 \%$ & L>L0@15 h & $\begin{array}{l}66.67 \mathrm{~mA} \mathrm{~cm}^{-} \\
2\end{array}$ \\
\hline $\mathrm{Cs} \mathrm{PbBr}_{3}: \mathrm{PEO}^{13}$ & ITO/NiOx/CsPbBr $3 / \mathrm{TPBi} / \mathrm{LiF} / \mathrm{Al}$ & $2.94 \%$ & $\begin{array}{l}\mathrm{L}>\mathrm{L}_{0} \text { after } 30 \\
\text { mins }\end{array}$ & $3.1 \mathrm{~V}$ \\
\hline
\end{tabular}




\begin{tabular}{|c|c|c|c|c|}
\hline $\mathrm{CsPbBr}: \mathrm{KBr}^{14}$ & ITO/p-TPD/PVK/Perov/TPBi/LiF/AI & $7.7 \%$ & $\mathrm{~T}_{50}=3.5$ hours & $\begin{array}{l}\text { Initial } \\
\text { luminance = } \\
100 \mathrm{~cd} \mathrm{~m}^{-2}\end{array}$ \\
\hline$B A B r: \mathrm{CsPbBr}_{3}{ }^{15}$ & $\begin{array}{l}\text { ITO/PEDOT:PSS/PVK/Perov/TPBi/ } \\
\text { LiF/AI }\end{array}$ & $10.10 \%$ & $\mathrm{t} 75=45 \mathrm{mins}$ & $\sim 26 \mathrm{~mA} \mathrm{~cm}^{-2}$ \\
\hline$N M A B r: C s P b B r{ }_{3}{ }^{16}$ & ITO/TFB/PVK/Perov/TPBi/LiF/AI & $4.70 \%$ & $\mathrm{t} 70=120 \mathrm{~s}$ & $0.2 \mathrm{~mA} \mathrm{~cm}^{-2}$ \\
\hline PEABr:CsPbBr ${ }_{3}^{17}$ & $\begin{array}{l}\text { ITO/poly-TPD/PFN/MHP/ } \\
\text { TPBi/LiF/AI }\end{array}$ & $15.50 \%$ & $\mathrm{t} 50=90 \mathrm{~min}$ & $2 \mathrm{~mA} \mathrm{~cm}^{-2}$ \\
\hline$B A B r: \mathrm{CsPbBr}_{3}{ }^{18}$ & $\begin{array}{l}\text { ITO/PEDOT:PSS/TFB/Perov/TPBi/ } \\
\text { Ca/Al }\end{array}$ & $8.42 \%$ & $\mathrm{t} 50=45 \mathrm{mins}$ & $3.4 \mathrm{~V}$ \\
\hline$P E A B r: \mathrm{CsPbBr}_{3}{ }^{14}$ & ITO/p-TPD/PVK/Perov/TPBi/LiF/AI & $16 \%$ & $\mathrm{~T}_{50}=0.8$ hours & $\begin{array}{l}\text { Initial } \\
\text { luminance }= \\
100 \mathrm{~cd} \mathrm{~m}^{-2}\end{array}=$ \\
\hline $\begin{array}{l}P E A B r+N a B r: C s P b B r \\
3^{19}\end{array}$ & ITO/NiOx/PVK/Perov/TPBi/LiF/AI & $12 \%$ & $\mathrm{~T}_{50}=40 \mathrm{mins}$ & $0.33 \mathrm{~mA} \mathrm{~cm}^{-2}$ \\
\hline $\mathrm{CsPbBr}_{3} \mathrm{NPs} 20$ & poly-TPD/PFI/Perov/TPBi/Li F/Al & $0.06 \%$ & $\mathrm{~L} 50 \approx 10 \mathrm{~min}$ & $5 \mathrm{~V}$ \\
\hline $\mathrm{CsPbBr}_{3} \mathrm{NPs}^{21}$ & $\begin{array}{l}\text { PEDOT:PSS/PTAA/MHP/TPBi/LiF/ } \\
\text { Al }\end{array}$ & $16.5 \%$ & $\mathrm{~L} 50=136 \mathrm{mins}$ & $0.6 \mathrm{~mA} \mathrm{~cm}-2$ \\
\hline
\end{tabular}

Table S2. Literature review of Stability and efficiency of state of the art $\mathrm{CsPbBr}_{3}$

perovskite LEDs. The results are in three categories: Blue $=$ Bulk $\mathrm{CsPbBr}_{3}$ with polymer or small molecule additives; Yellow $=$ Alkylammonium additives with $\mathrm{CsPbBr}_{3}$ spincoated together; Green $=$ colloidal $\mathrm{CsPbBr}_{3}$ nanocrystals which are coordinated with ammonium salts. 


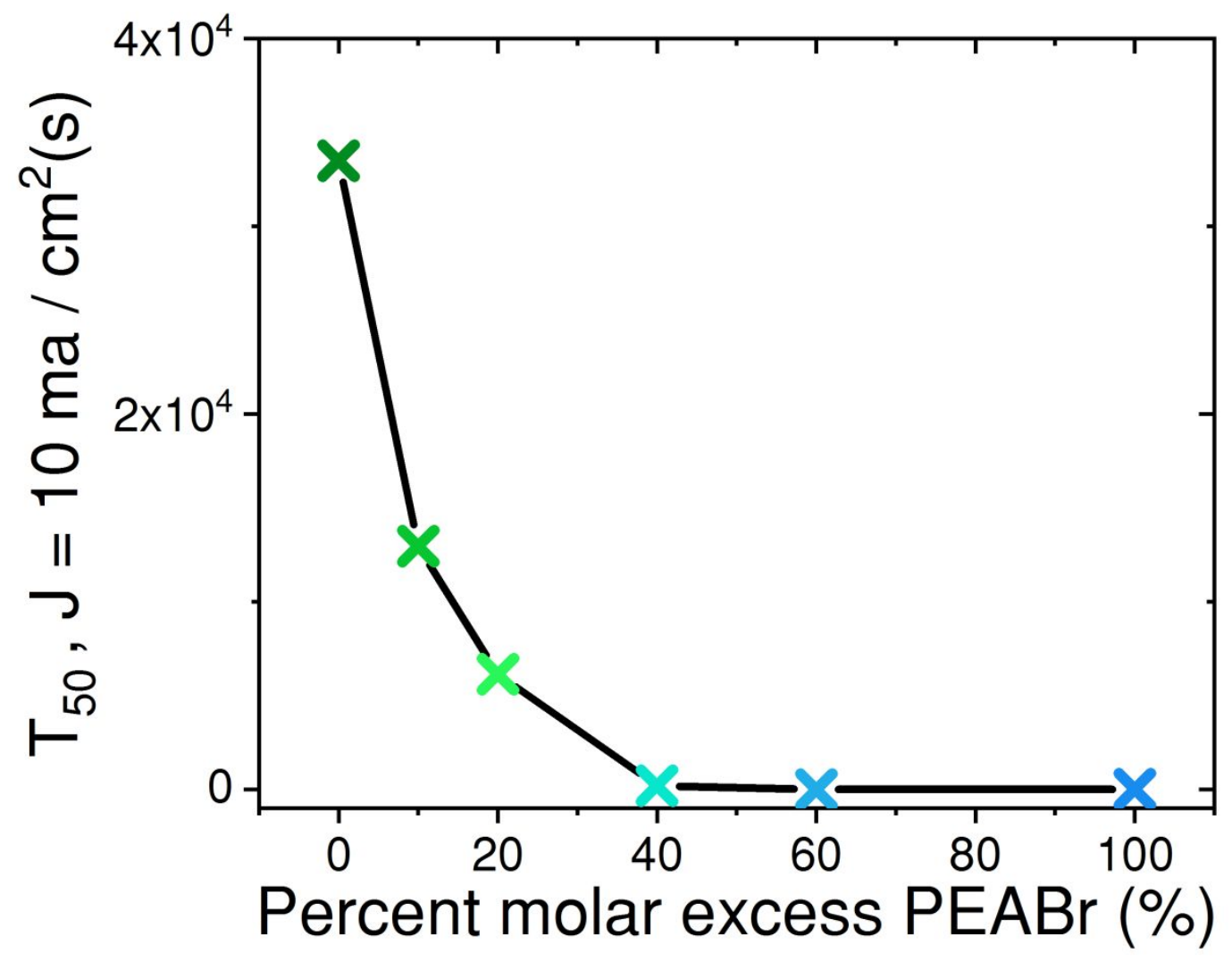

Figure S6. Graphical plot of $\mathrm{T}_{50}$ vs $\mathrm{PEABr}$ amount showing the strong decrease in stability when $\mathrm{PEABr}$ is added. 


\begin{tabular}{|c|c|c|}
\hline Perovskite Composition & 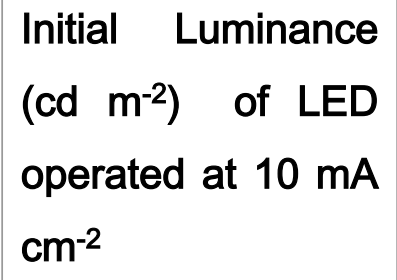 & $\begin{array}{l}\text { Peak Luminance (cd m- } \\
\text { 2) of LED operated at } 10 \\
\mathrm{~mA} \mathrm{~cm}-2\end{array}$ \\
\hline $\mathrm{CsPbBr}_{3}$ & 14 & 61 \\
\hline$(\mathrm{PEABr})_{0.1} \mathrm{CsPbBr}_{3}$ & 58 & 91 \\
\hline$(\mathrm{PEABr})_{0.2} \mathrm{CsPbBr}_{3}$ & 266 & 271 \\
\hline$(\mathrm{PEABr})_{0.4} \mathrm{CsPbBr}_{3}$ & 3427 & 3437 \\
\hline$(\mathrm{PEABr})_{0.6} \mathrm{CsPbBr}_{3}$ & 278 & 307 \\
\hline$(\mathrm{PEABr})_{1.0} \mathrm{CsPbBr}_{3}$ & 16 & 19 \\
\hline
\end{tabular}

Table S3. Initial and peak luminance of perovskite LEDs when operated at $10 \mathrm{~mA} \mathrm{~cm}^{-2}$.

Note

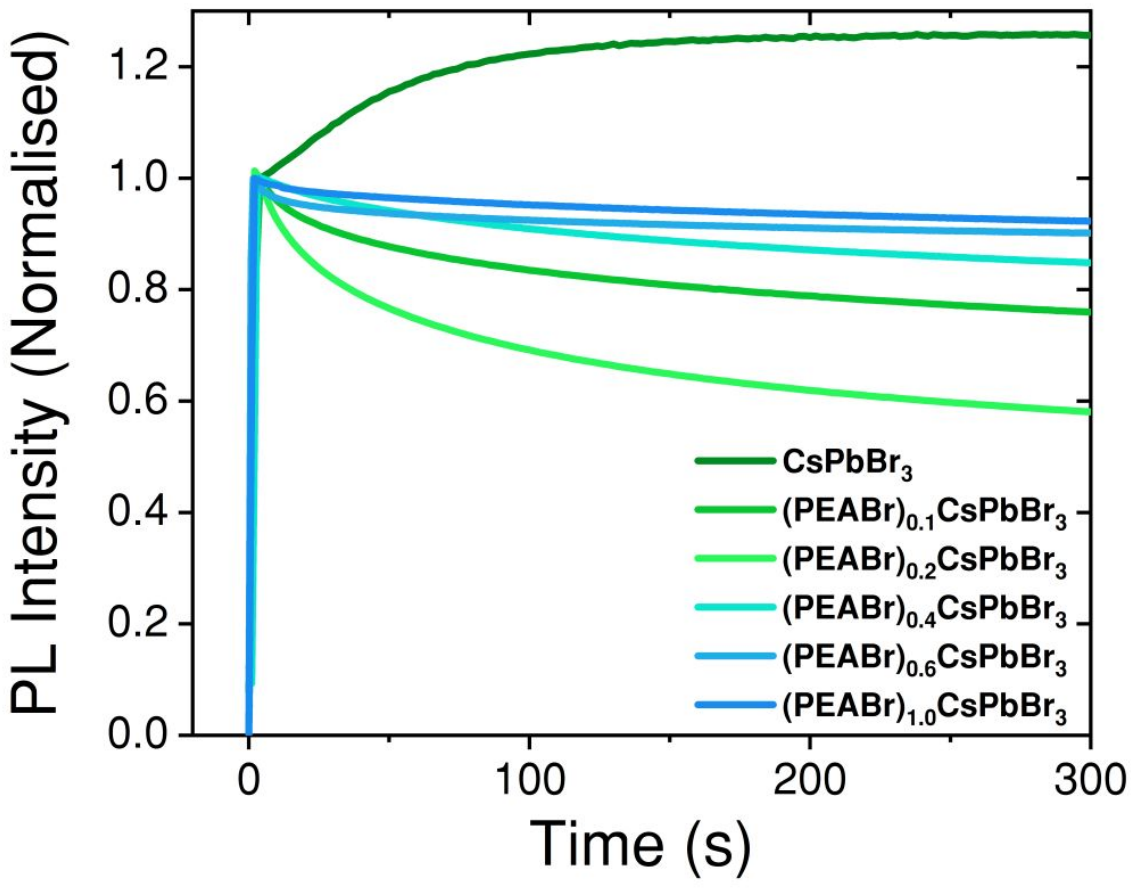

that

some

LEDs

brighten

during

operation at constant current density. 
Figure S7. Photostability as a function of composition at an irradiance of $93 \mathrm{~mW} / \mathrm{cm}^{2}$ $; \lambda_{\mathrm{exc}}=405 \mathrm{~nm}$. All samples were encapsulated to remove the effects of atmosphere on the measurement. One can see that excluding $\mathrm{CsPbBr}_{3}$ the sample with the best photostability are $(\mathrm{PEABr})_{0.6} \mathrm{CsPbBr}_{3}$ and $(\mathrm{PEABr})_{1.0} \mathrm{CsPbBr}_{3}$ which had the worst $\mathrm{EQE}_{\mathrm{EL}}$ stability 


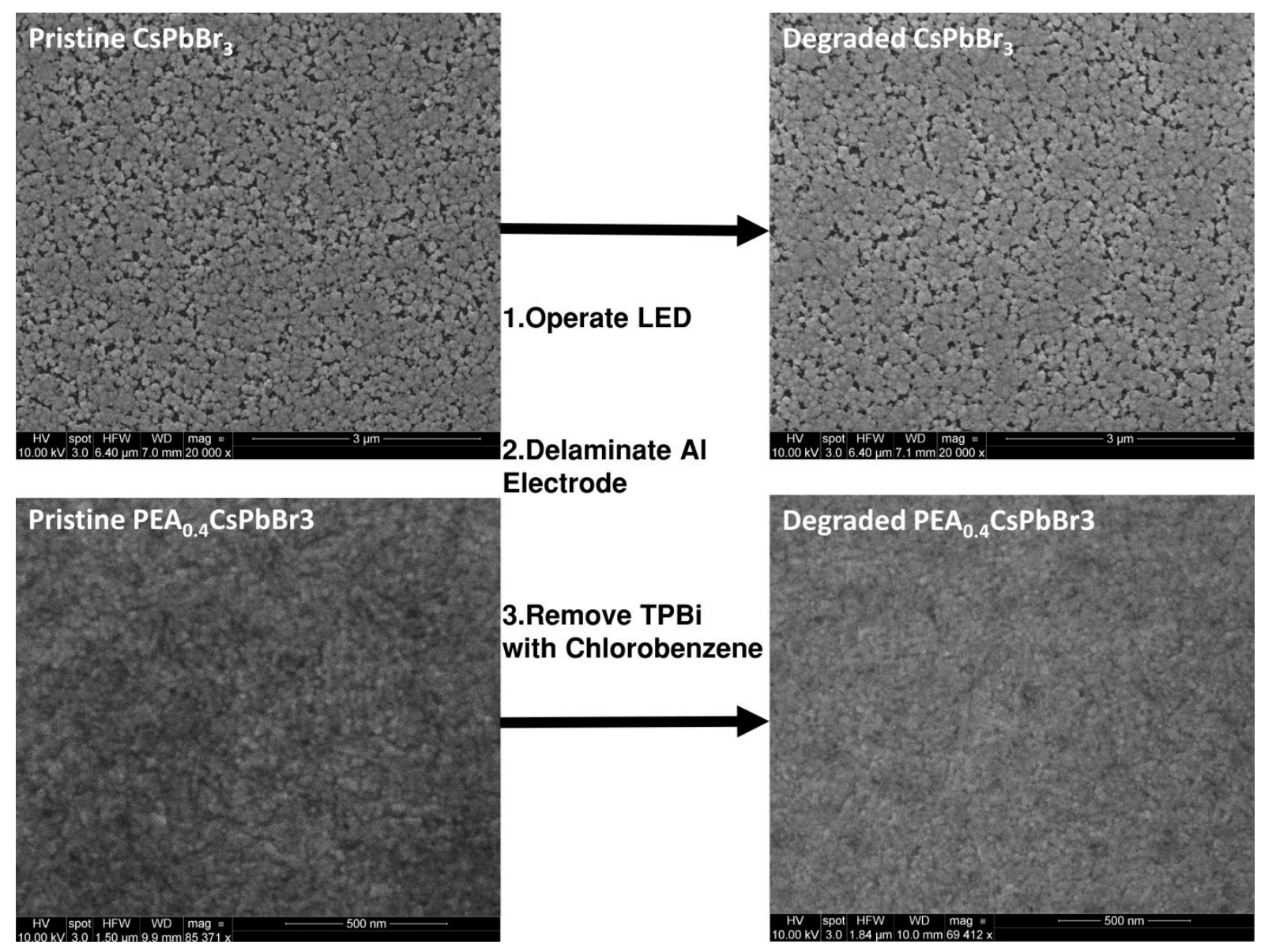

Figure S8. SEM characterisation of our control films and our most efficient composition

$(\mathrm{PEABr})_{0.4} \mathrm{CsPbBr}_{3}$ before and after operation. The samples were operated and then

the Al electrode was delaminated and the TPBi electron injection layer was washed

off with chlorobenzene 

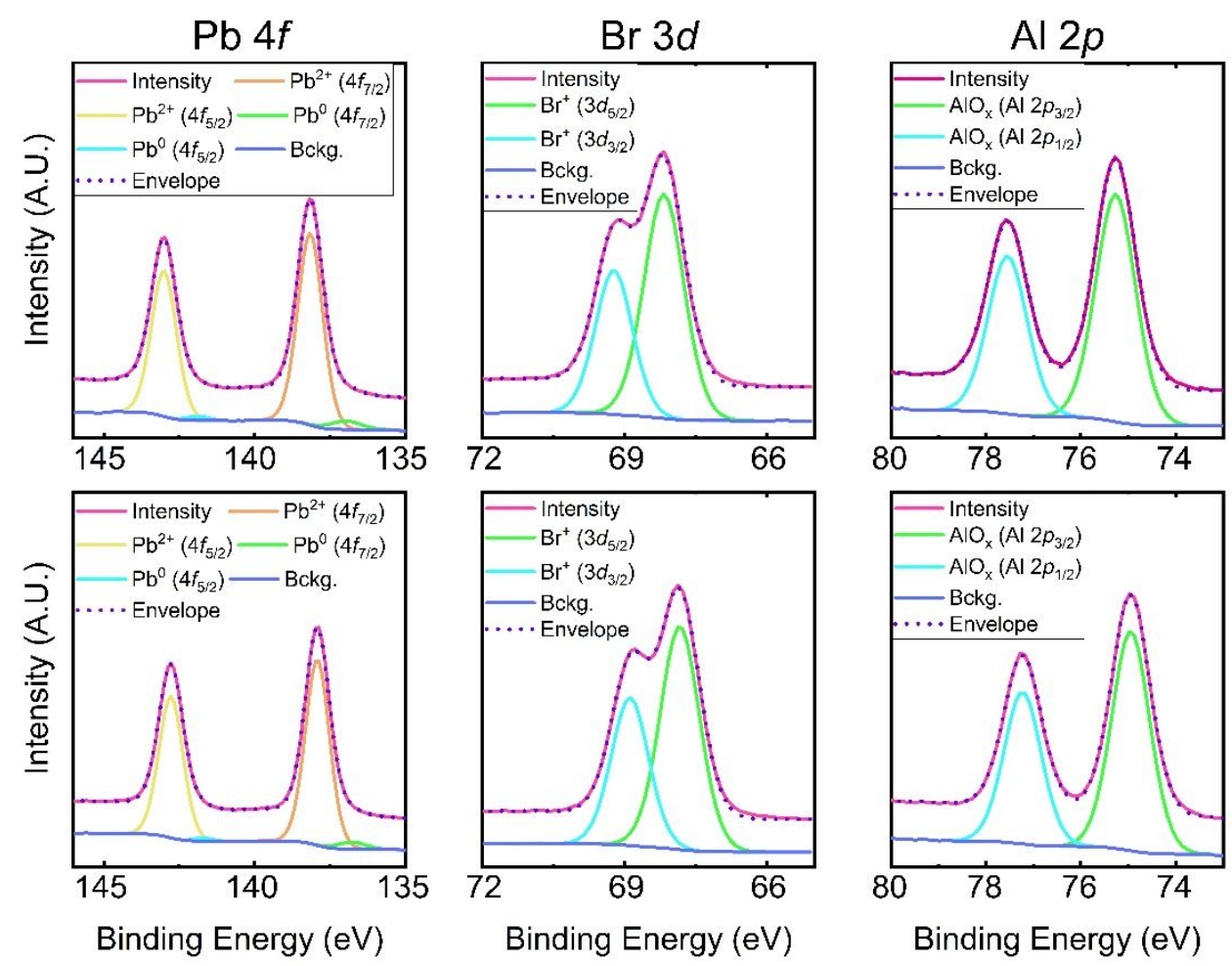

Figure S9. High resolution XPS spectra of $\mathrm{Pb} 4 \mathrm{f}, \mathrm{Br} 3 \mathrm{~d}$ and $\mathrm{Al} 2 \mathrm{p}$ for unoperated LED devices. Top row shows the control $\mathrm{CsPbBr}_{3}$ devices and bottom row shows those with $(\mathrm{PEABr})_{0.4} \mathrm{CsPbBr}$. Spectra were fit using the Casa XPS software, peaks were fit using a mixture of Gaussian and Lorentzian (Lorentzian $=30 \%$ ) line shapes using a Shirley background. Devices were fabricated, the Al electrode was delaminated and the TPBi electron injection layer was washed off with chlorobenzene. The resulting samples were those measured here. 

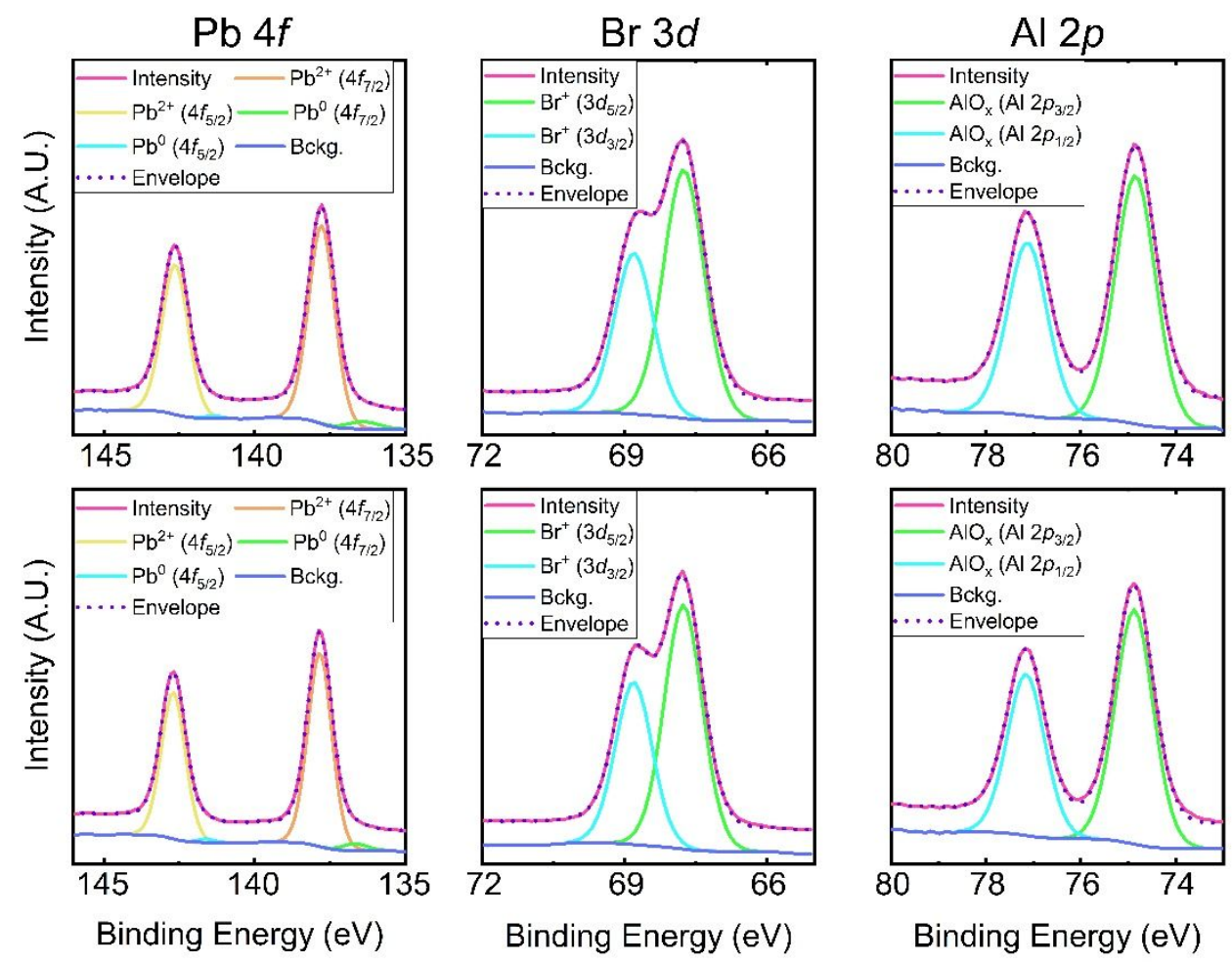

Figure S10. High resolution XPS spectra of $\mathrm{Pb} 4 \mathrm{f}, \mathrm{Br} 3 \mathrm{~d}$ and $\mathrm{Al} 2 \mathrm{p}$ for operated LED devices. Top row shows the control $\mathrm{CsPbBr}_{3}$ devices and bottom row shows those with $(\mathrm{PEABr})_{0.4} \mathrm{CsPbBr}$. Spectra were fit using the Casa XPS software, peaks were fit using a mixture of Gaussian and Lorentzian (Lorentzian $=30 \%$ line shapes using a Shirley background. Devices were fabricated, the Al electrode was delaminated and the TPBi electron injection layer was washed off with chlorobenzene. The resulting samples were those measured here. 
Unop. control

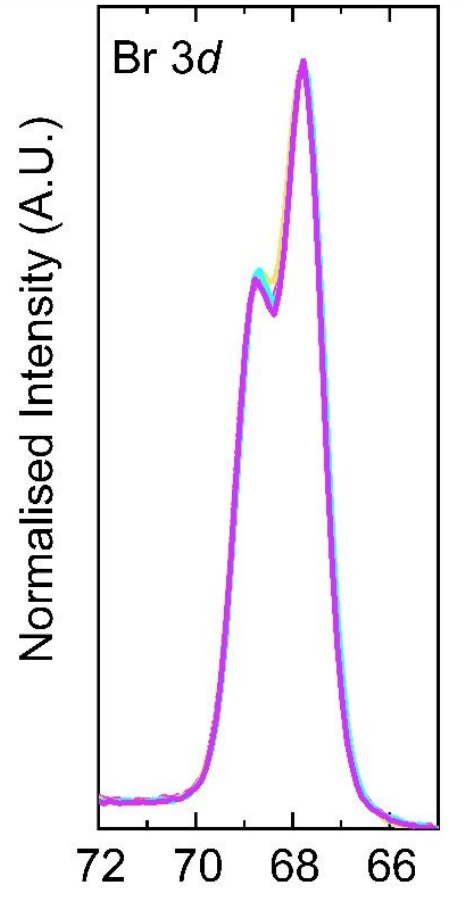

Op. control

$\mathrm{Pb} 4 f$

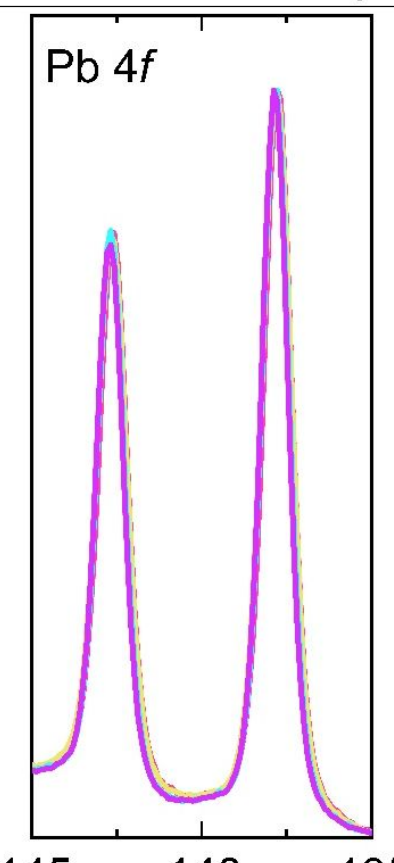

145
Unop. PEABr

Op. PEABr

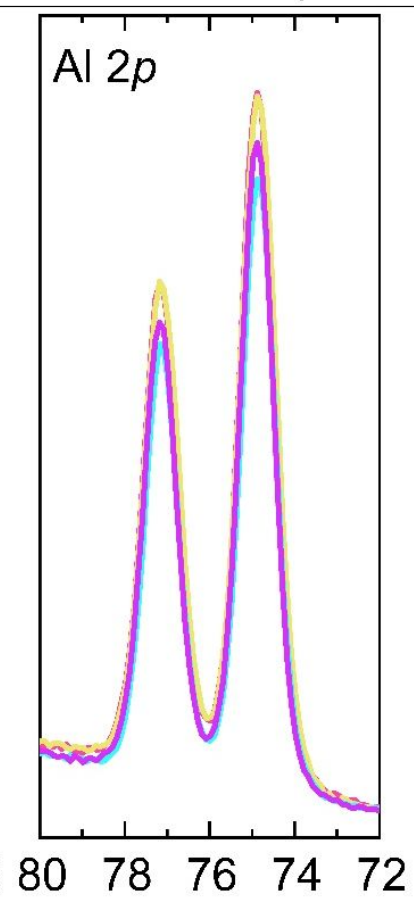

Binding Energy (eV)

Figure S11. Overlapped high resolution XPS spectra of $\mathrm{Pb} 4 \mathrm{f}, \mathrm{Br} 3 \mathrm{~d}$ and $\mathrm{Al} 2 \mathrm{p}$ for unoperated (unop.) and operated (op.) LED devices. PEABr concentration in the relevant devices is $40 \%$ molar excess. Figure S9 shows no significant shift in peak positions between control and $\mathrm{PEABr}$ devices and those which have been operated vs unoperated. 


\begin{tabular}{|l|l|l|l|}
\hline $\begin{array}{l}\text { Control } \\
\text { unoperated }\end{array}$ & 67.8 & 137.8 & 74.9 \\
\hline Control operated & 67.8 & 137.8 & 74.9 \\
\hline PEABr unoperated & 67.8 & 137.8 & 74.9 \\
\hline PEABr operated & 67.8 & 137.9 & 74.9 \\
\hline
\end{tabular}

Table S4. Peak positions for all samples measured using XPS
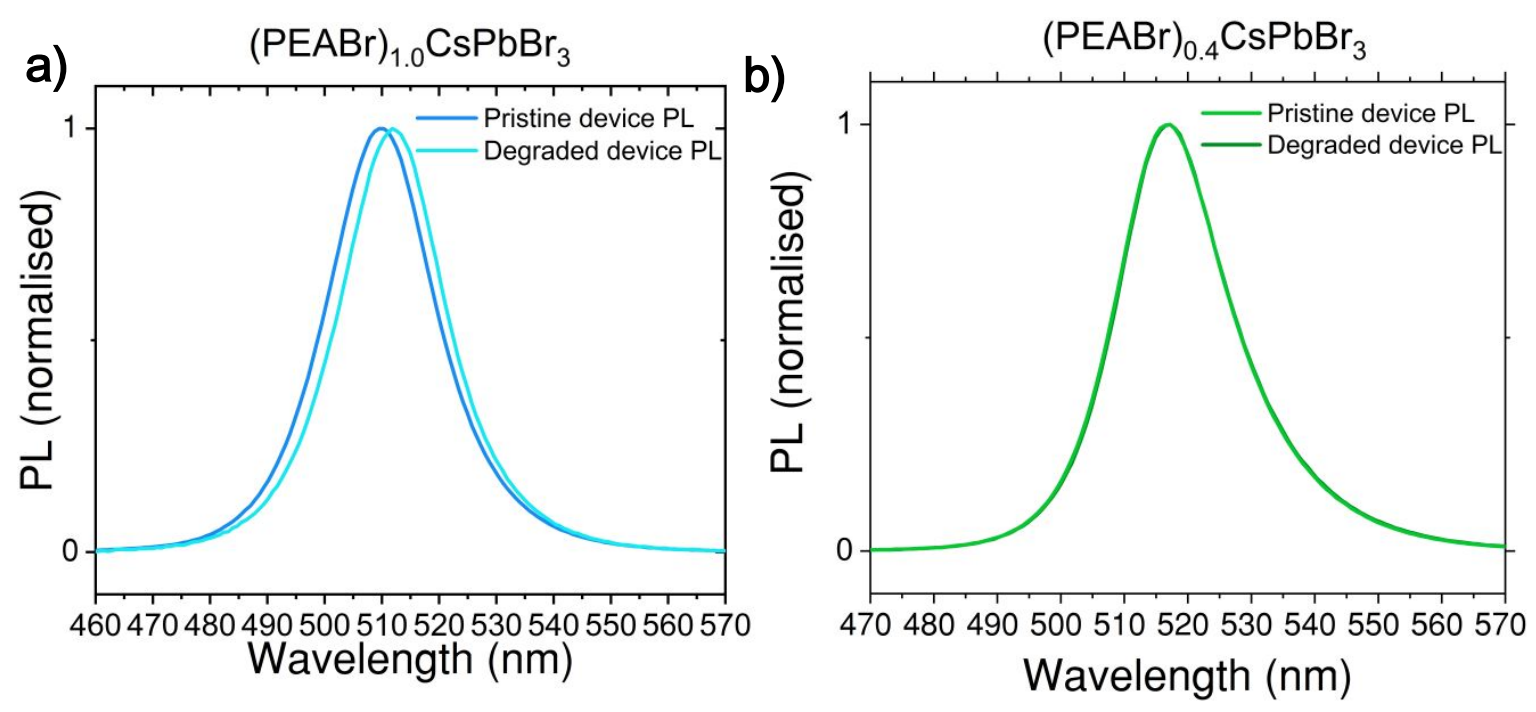

Figure S12. Photoluminescence of $(\mathrm{PEABr})_{1.0} \mathrm{CsPbBr}_{3}$ and $(\mathrm{PEABr})_{0.4} \mathrm{CsPbBr}{ }_{3}$ device before and after operation for $1 \mathrm{~h}$ at $10 \mathrm{~mA} / \mathrm{cm}^{2}$ 

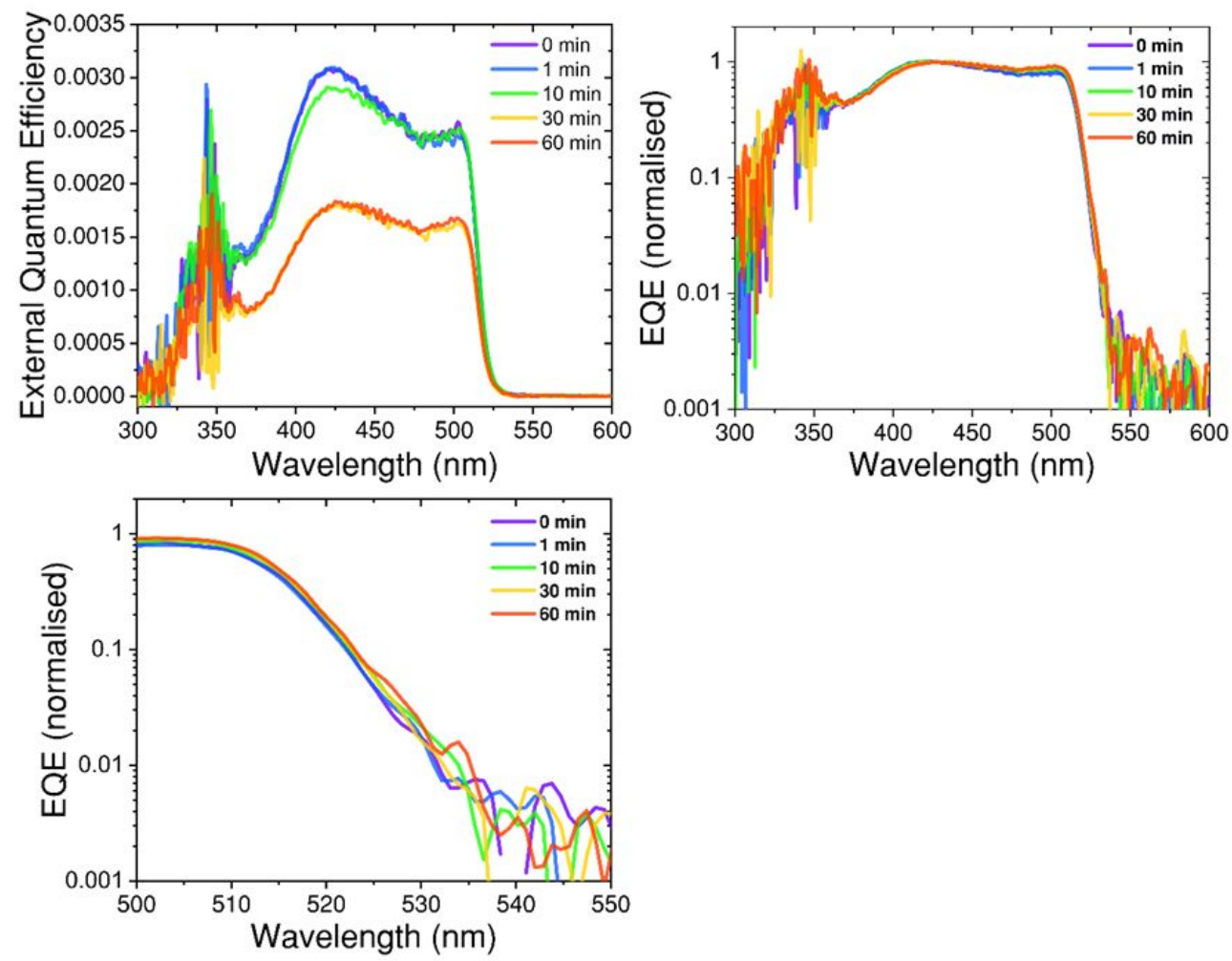

Figure S13. Fourier transform photocurrent spectroscopy data plotted as external quantum efficiency. The data is shown on absolute and normalised scales. A zoomed in plot of the band tail shows no discernible changes. 
a)

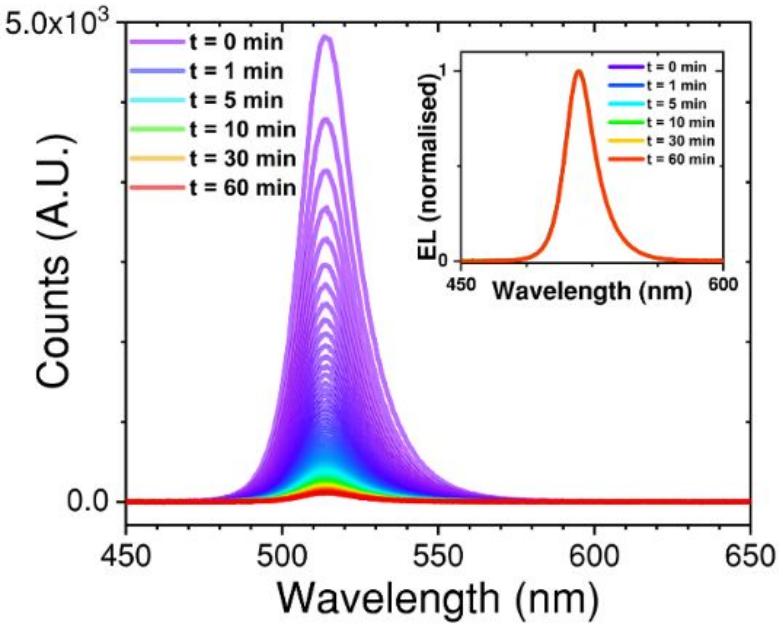

c)

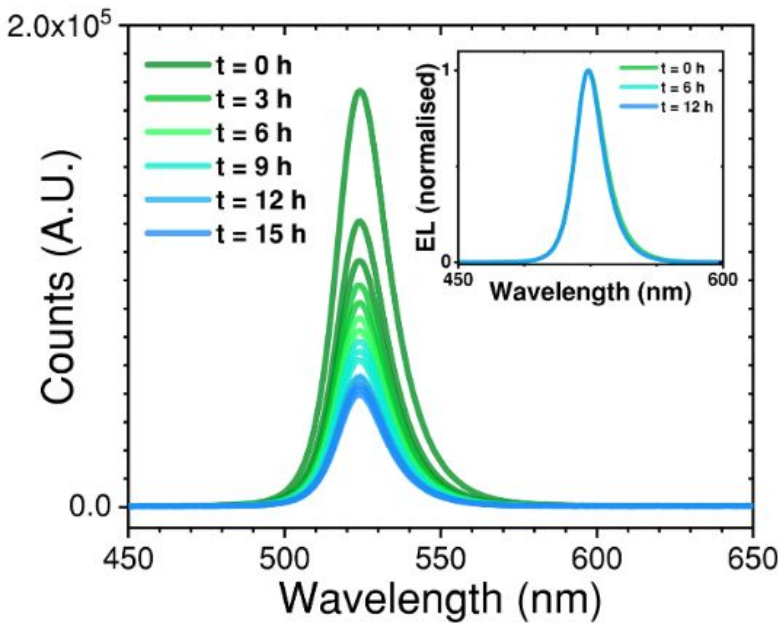

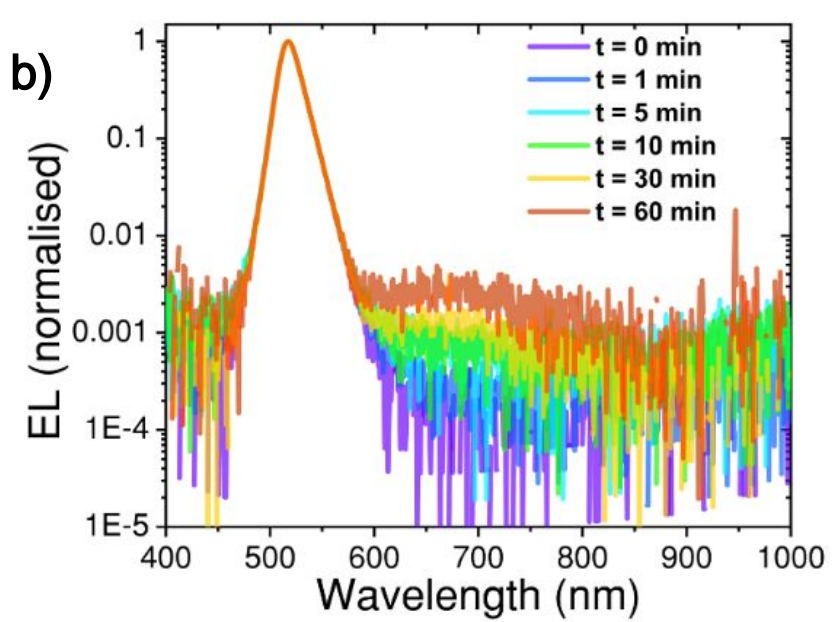

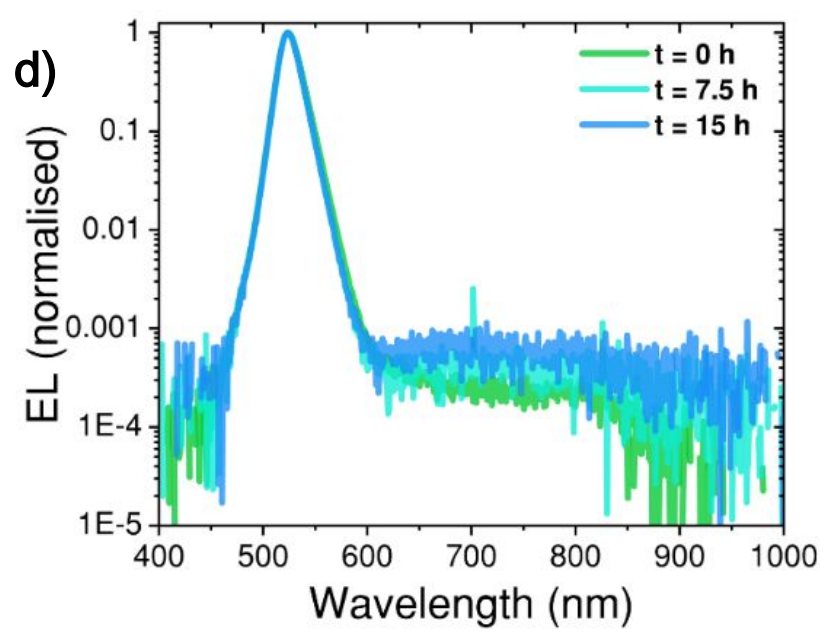

Figure S14. Evidence of emissive trap states in $\mathrm{CsPbBr}_{3}$ LEDs: a) Decay of intensity of EL for $(\mathrm{PEABr})_{0.4} \mathrm{CsPbBr}_{3} \mathrm{LEDs}$ operated at $20 \mathrm{~mA} / \mathrm{cm}^{2}$ on an absolute scale (inset: The same data on a normalised scale); b) Normalised EL spectra of $(\mathrm{PEABr})_{0.4} \mathrm{CsPbBr}_{3} \mathrm{LEDs}$ operated at $20 \mathrm{~mA} / \mathrm{cm}^{2}$ on a log scale showing the growth of a broad shoulder in the emission; c) Decay of intensity of $\mathrm{EL} \mathrm{CsPbBr}_{3} \mathrm{LEDs}$ operated at $20 \mathrm{~mA} / \mathrm{cm}^{2}$ on an absolute scale (inset: The same data on a normalised scale), note the remarkably superior stability compared with $\left.(\mathrm{PEABr})_{0.4} \mathrm{CsPbBr}_{3} ; b\right)$ Normalised $\mathrm{EL}$ 
spectra of $\mathrm{CsPbBr}_{3}$ LEDs operated at $20 \mathrm{~mA} / \mathrm{cm}^{2}$ on a log scale showing the growth of a broad shoulder in the emission, note that the shoulder is present in the pristine device whereas it is not in $(\mathrm{PEABr})_{0.4} \mathrm{CsPbBr}_{3}$. 
The following script can be imported into python to calculate the radiation view factor of two coaxial, parallel square plates:"'"'

Radiation View factors

https://en.wikipedia.org/wiki/View_factor

http://webserver.dmt.upm.es/ isidoro/tc3/Radiation\%20View\%20factors.pdf (Page 24 square coaxial plates)

@update: 11/3/2019

@author: wenger

"'"

import numpy as $\mathrm{np}$

$\mathrm{W} 1=3$

$\mathrm{W} 2=10$

$\mathrm{H}=9$

def $\mathrm{CF}(\mathrm{w} 1, \mathrm{w} 2, \mathrm{H})$ :

$\mathrm{w} 1=\mathrm{W} 1 / \mathrm{H}$

$\mathrm{w} 2=\mathrm{W} 2 / \mathrm{H}$

$\mathrm{x}=\mathrm{w} 2-\mathrm{w} 1$

$y=w 2+w 1$

$p=\left(w 1^{* *} 2+w 2^{* *} 2+2\right)^{* *} 2$

$q=\left(x^{* *} 2+2\right) *\left(y^{* *} 2+2\right)$

$u=n p . \operatorname{sqrt}\left(x^{* *} 2+4\right)$

$v=\operatorname{np} . \operatorname{sqrt}\left(\mathrm{y}^{* *} 2+4\right)$

$s=u^{*}\left(x{ }^{*} n p \cdot \arctan (x / u)-y{ }^{*} n p \cdot \arctan (y / u)\right)$

$\mathrm{t}=\mathrm{v}^{*}\left(\mathrm{x}{ }^{*} \mathrm{np} \cdot \arctan (\mathrm{x} / \mathrm{v})-\mathrm{y}{ }^{*} \mathrm{np} \cdot \arctan (\mathrm{y} / \mathrm{v})\right)$

$F 12=1 /\left(n p \cdot p i^{*} w 1^{* *} 2\right){ }^{*}(n p \cdot \log (p / q)+s-t)$

print('the estimated value is',(w1*(W2/W1) $)^{\star *} 2$ / np.pi)

return F12 
$\mathrm{F} 12=\mathrm{CF}(\mathrm{W} 1, \mathrm{~W} 2, \mathrm{H})$

print('The calculated value is',F12)

Figure S15. Script for calculation of Radiation view factor for Lambertian emission from square plate (i.e. the LED) to a coaxial square plate (i.e. the photodiode)

\section{References}

(1) Hancock, R. D.; Martell, A. E. The Chelate, Cryptate and Macrocyclic Effects. Comments Inorg. Chem. 1988, 6, 237-284.

(2) Rüdiger, V.; Schneider, H.-J.; Solov'ev, V. P.; Kazachenko, V. P.; Raevsky, O. A. Crown Ether-Ammonium Complexes: Binding Mechanisms and Solvent Effects. European J. Org. Chem. 1999, 8, 1847-1856.

(3) Goff, C. M.; Matchette, M. A.; Shabestary, N.; Khazaeli, S. Complexation of Caesium and Rubidium Cations with Crown Ethers in N,N-Dimethylformamide. Polyhedron 1996, 15, 3897-3903.

(4) Nayak, P. K.; Sendner, M.; Wenger, B.; Wang, Z.; Sharma, K.; Ramadan, A. J.; Lovrinčić, R.; Pucci, A.; Madhu, P. K.; Snaith, H. J. Impact of $\mathrm{Bi}^{3+}$ Heterovalent Doping in OrganicInorganic Metal Halide Perovskite Crystals. J. Am. Chem. Soc. 2018, 140, 574-577.

(5) Ulatowski, A. M.; Wright, A. D.; Wenger, B.; Buizza, L. R. V.; Motti, S. G.; Eggimann, H. J.; Savill, K. J.; Borchert, J.; Snaith, H. J.; Johnston, M. B.; Herz, L. M. Charge-Carrier Trapping Dynamics in Bismuth-Doped Thin Films of $\mathrm{MAPbBr}_{3}$ Perovskite. J. Phys. Chem. Lett. 2020, 11, 3681-3688.

(6) Wang, H.; Zhang, X.; Wu, Q.; Cao, F.; Yang, D.; Shang, Y.; Ning, Z.; Zhang, W.; Zheng, W.; Yan, Y.; Kershaw, S. V.; Zhang, L.; Rogach, A. L.; Yang, X. Trifluoroacetate Induced SmallGrained $\mathrm{CsPbBr}_{3}$ Perovskite Films Result in Efficient and Stable Light-Emitting Devices. Nat. Commun. 2019, 10, 665.

(7) Hu, Y.; Wang, Q.; Shi, Y.-L.; Li, M.; Zhang, L.; Wang, Z.-K.; Liao, L.-S. Vacuum-Evaporated All-Inorganic Cesium Lead Bromine Perovskites for High-Performance Light-Emitting Diodes. J. Mater. Chem. C. 2017, 5, 8144-8149.

(8) Qin, C.; Matsushima, T.; Sandanayaka, A. S. D.; Tsuchiya, Y.; Adachi, C. CentrifugalCoated Quasi-Two-Dimensional Perovskite $\mathrm{CsPb}_{2} \mathrm{Br}_{5}$ Films for Efficient and Stable Light- 
Emitting Diodes. J. Phys. Chem. Lett. 2017, 8, 5415-5421.

(9) Wu, T.; Li, J.; Zou, Y.; Xu, H.; Wen, K.; Wan, S.; Bai, S.; Song, T.; McLeod, J. A.; Duhm, S.; Gao, F.; Sun, B. High-Performance Perovskite Light-Emitting Diode with Enhanced Operational Stability Using Lithium Halide Passivation. Angew. Chemie Int. Ed. 2020, $59,4099-4105$

(10) Wu, C.; Zou, Y.; Wu, T.; Ban, M.; Pecunia, V.; Han, Y.; Liu, Q.; Song, T.; Duhm, S.; Sun, B. Improved Performance and Stability of All-Inorganic Perovskite Light-Emitting Diodes by Antisolvent Vapor Treatment. Adv. Funct. Mater. 2017, 2, 1-7.

(11) Cho, H.; Wolf, C.; Kim, J. S.; Yun, H. J.; Bae, J. S.; Kim, H.; Heo, J.-M.; Ahn, S.; Lee, T.-W. High-Efficiency Solution-Processed Inorganic Metal Halide Perovskite Light-Emitting Diodes. Adv. Mater. 2017, 29, 1700579.

(12) Wei, Z.; Perumal, A.; Su, R.; Sushant, S.; Xing, J.; Zhang, Q.; Tan, S. T.; Demir, H. V.; Xiong, Q. Solution-Processed Highly Bright and Durable Cesium Lead Halide Perovskite LightEmitting Diodes. Nanoscale 2016, 8, 18021-18026.

(13) Wang, Z.; Luo, Z.; Zhao, C.; Guo, Q.; Wang, Y.; Wang, F.; Bian, X.; Alsaedi, A.; Hayat, T.; Tan, Z. Efficient and Stable Pure Green All-Inorganic Perovskite CsPbBr ${ }_{3}$ Light-Emitting Diodes with a Solution-Processed NiOx Interlayer. J. Phys. Chem. C 2017, 121, 2813228138.

(14) Zou, C.; Liu, Y.; Ginger, D. S.; Lin, L. Y. Suppressing Efficiency Roll-Off at High Current Densities for Ultra-Bright Green Perovskite Light-Emitting Diodes. ACS Nano 2020, 14 , 6076-6086.

(15) Vashishtha, P.; Ng, M.; Shivarudraiah, S. B.; Halpert, J. E. High Efficiency Blue and Green Light-Emitting Diodes Using Ruddlesden-Popper Inorganic Mixed Halide Perovskites with Butylammonium Interlayers. Chem. Mater. 2019, 31, 83-89.

(16) Wu, T.; Yang, Y.; Zou, Y.; Wang, Y.; Wu, C.; Han, Y.; Song, T.; Zhang, Q.; Gao, X.; Sun, B. Nanoplatelet Modulation in 2D/3D Perovskite Targeting Efficient Light-Emitting Diodes. Nanoscale 2018, 10, 19322-19329.

(17) Ban, M.; Zou, Y.; Rivett, J. P. H.; Yang, Y.; Thomas, T. H.; Tan, Y.; Song, T.; Gao, X.; Credgington, D.; Deschler, F.; Sirringhaus, H.; Sun, B. Solution-Processed Perovskite Light Emitting Diodes with Efficiency Exceeding 15\% through Additive-Controlled Nanostructure Tailoring. Nat. Commun. 2018, 9, 3892.

(18) Wang, Z.; Wang, F.; Sun, W.; Ni, R.; Hu, S.; Liu, J.; Zhang, B.; Alsaed, A.; Hayat, T.; Tan, Z. Manipulating the Trade-Off between Quantum Yield and Electrical Conductivity for High-Brightness Quasi-2D Perovskite Light-Emitting Diodes. Adv. Funct. Mater. 2018, 28, 1804187.

(19) Chen, H.; Fan, L.; Zhang, R.; Liu, W.; Zhang, Q.; Guo, R.; Zhuang, S.; Wang, L. Sodium Ion Modifying In Situ Fabricated $\mathrm{CsPbBr}_{3}$ Nanoparticles for Efficient Perovskite Light Emitting Diodes. Adv. Opt. Mater. 2019, 7, 1900747.

(20) Zhang, X.; Lin, H.; Huang, H.; Reckmeier, C.; Zhang, Y.; Choy, W. C. H.; Rogach, A. L. 
Enhancing the Brightness of Cesium Lead Halide Perovskite Nanocrystal Based Green Light-Emitting Devices through the Interface Engineering with Perfluorinated Ionomer. Nano Lett. 2016, 16, 1415-1420.

(21) Song, J.; Fang, T.; Li, J.; Xu, L.; Zhang, F.; Han, B.; Shan, Q.; Zeng, H. Organic-Inorganic Hybrid Passivation Enables Perovskite QLEDs with an EQE of $16.48 \%$. Adv. Mater. 2018, 30, 1805409. 